\title{
Exosomes derived from siRNA against GRP78 modified bone-marrow-derived mesenchymal stem cells suppress Sorafenib resistance in hepatocellular carcinoma
}

\author{
Hongdan $\mathrm{Li}^{1^{*}}$, Cheng Yang ${ }^{2}$, Yijie Shi ${ }^{3}$ and Liang Zhao ${ }^{3^{*}}$
}

\begin{abstract}
Background: Sorafenib is an effective clinical drug in therapy of hepatocellular carcinoma, having led to improved prognosis in hepatocellular carcinoma patients. However acquired resistance is still being encountered. So, it is urgently to develop alternative strategies to overcome drug resistance. Exosomes can be modified with a variety of molecules, thereby acting as a vehicle for the delivery of therapeutic agents. The GRP78 is overexpressed in Sorafenib resistant cancer cells compared to Sorafenib sensitive cancer cells and thus is able to act as a target for therapy of hepatocellular carcinoma.

Results: In this study, we modified BM-MSCs to express the exosomal siGRP78. And we show that siGRP78 modified exosomes combined with Sorafenib is able to target GRP78 in hepatocellular carcinoma cells and inhibit the growth and invasion of the cancer cells in vitro. Further, siGRP78 modified exosomes combined with Sorafenib also inhibit the growth and metastasis of the cancer cells in vivo.
\end{abstract}

Conclusions: siGRP78 modified exosomes could sensitize Sorafenib resistant cancer cells to Sorafenib and reverse the drug resistance.

Keywords: Exosome, GRP78, Sorafenib, Growth, Metastasis, Hepatocellular carcinoma

\section{Background}

Hepatocellular carcinoma (HCC) is the sixth most common tumor and the second most frequent cause of cancer death worldwide [1, 2]. Nowadays, HCC presents a high incidence and mortality. Although many treatment have improved and diagnostic standardization has been better [3], improved overall survival of patients is difficult.

Sorafenib [4] is an oral multikinase inhibitor which inhibits HCC proliferation and increases apoptosis by inhibiting the serine-threonine kinases BRAF and CRAF and the receptor tyrosine kinases vascular endothelial

\footnotetext{
*Correspondence: lihongdan101@126.com; liangzhao79@163.com

${ }^{1}$ Life Science Institute, Jinzhou Medical University, Jinzhou 121000,

People's Republic of China

${ }^{3}$ School of Pharmacy, Jinzhou Medical University, Jinzhou 121000,

People's Republic of China

Full list of author information is available at the end of the article
}

growth factors receptors (VEGFRs) and platelet-derived growth factor receptor $\beta$ (PDGFR- $\beta$ ) [5]. Until now, Sorafenib is still the only FDA approved systemic drug for the treatment of unresectable advanced HCC. However, acquired resistance to Sorafenib in HCC patients is a common phenomenon and limits its clinical application [6-8].

Grp78 is overexpressed in many tumors and has been linked to the progression of many human cancers including colon cancer [9], lung cancer [10], gastric cancer [11], breast cancer [12], Hepatocellular carcinoma [13]. Our research group not only find GRP78 play important roles in HCC, but also find GRP78 promotes the drug resistance to Sorafenib [5, 14]. As a strategy for targeting drug resistance, the application of nucleic acid-based inhibitors of gene expression, such as RNA interference (RNAi), has been proposed in the 
treatment of many tumors [15-19]. And with the development of exosomes, researchers find exosomes is a therapeutic approach to delivery siRNA and some other factors [20, 21].

Exosomes are small nanometer-sized $(40-100 \mathrm{~nm})$ vesicles of endocytic origin. They are initially formed within the endosomal compartment and, subsequently secreted when a multi-vesicular body (MVB) fuses with the plasma membrane $[22,23]$. These vesicles are released by any type of cells including cancer cells [24]. It was recently reported that exosomes also contain siRNA and microRNA that are transferred to target cancer cells, where they can be translated or mediate RNA silencing $[25,26]$. In intercellular communication, exosomes have been considered messengers. Furthermore, exosomes have a complex protein membrane composition that contributes to efficient cellular uptake [27, 28]. Exosomes are an extremely promising therapeutic tool for numerous diseases given their ability to shuttle small molecules between cells. In particular, exosomes avoid immune recognition and clearance compared to exogenous nanovesicles [23]. They have been used widely, such as diabetes [29], cartilage tissue regeneration [30], stroke [31], tumors [32] and et al.
At present, many researchers used bone marrow mesenchymal stem cells (BM-MSCs) as a tool to gain modified exosomes for its low immunogens [33]. In this study, we generated modified BM-MSCs derived exosomes able to deliver GRP78 siRNA to hepatocellular carcinoma cells to overcome pharmacological resistance of Sorafenib (Fig. 1).

\section{Results}

\section{Characterization of siRNA against GRP78 modified}

\section{BM-MSCs}

To produce siGRP78 expressing exosomes, we isolated BM-MSCs (Fig. 2a) and transfected with siGRP78 or control siRNA into BM-MSCs (Fig. 2b). Then, we identified the expression of GRP78 in these cells expressed siGRP78 as shown by qPCR analysis. The flow cytometer results showed that GRP78 did not influence the stemness trait of BM-MSCs (Fig. 2c). qPCR results showed GRP78 was down-regulated in the siGRP78 transfected BM-MSCs (Fig. 2d).

\section{Characterization of Sorafenib resistant HCC cells}

To establish Sorafenib resistant cancer cells, we exposed HCC cells HepG2 and PLC to increasing concentrations
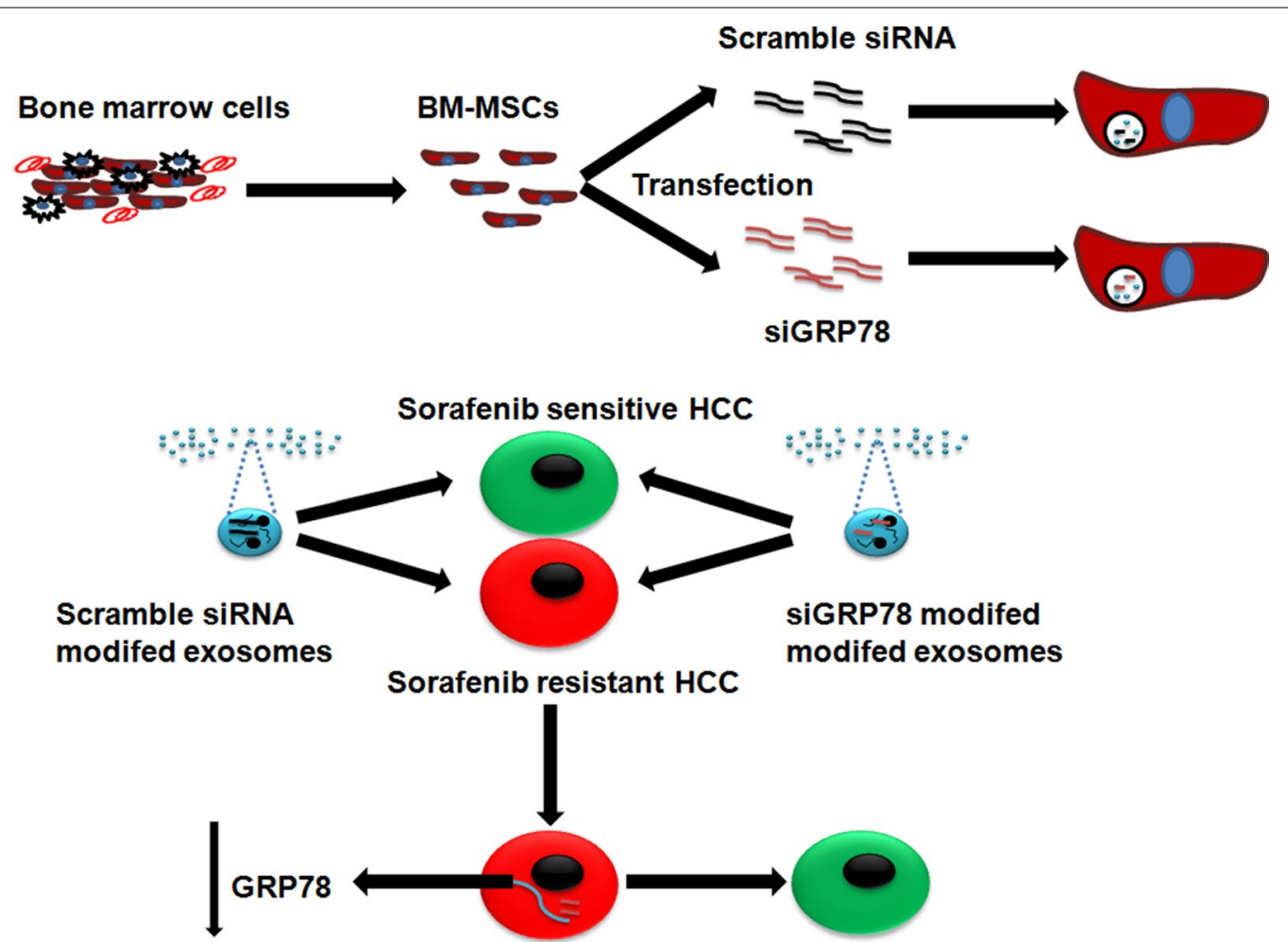

Fig. 1 Schematic representation of exosomes derived from BM-MSCs. BM-MSCs were transfected with scramble siRNA and siGRP78 to generate scramble siRNA modified exosomes and siGRP78 modified exosomes. The effect of modified exosomes were tested on Sorafenib sensitive or resistant HCC cells 


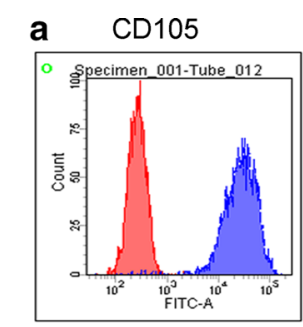

b
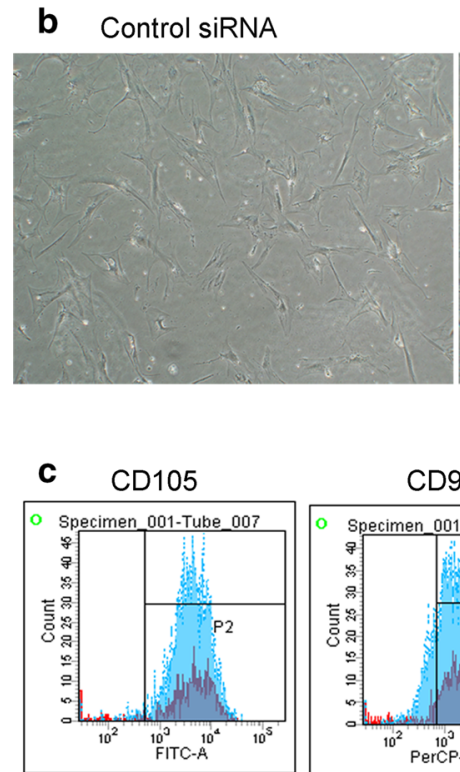
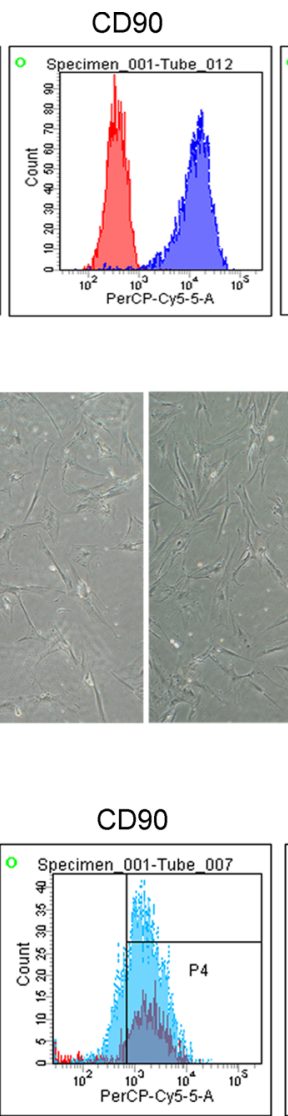

siGRP78

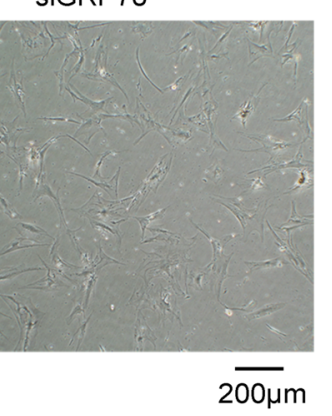

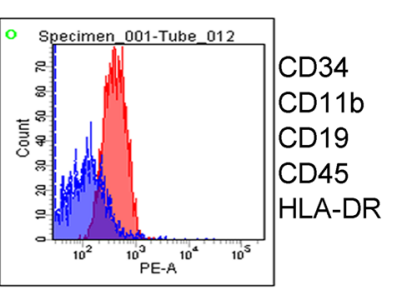

d
GRP78

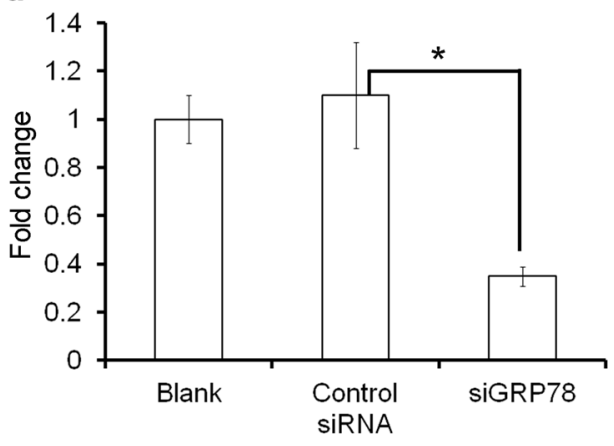

CD73

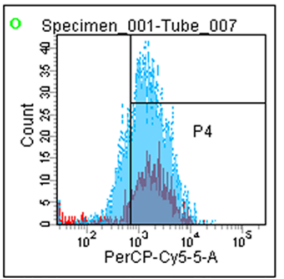

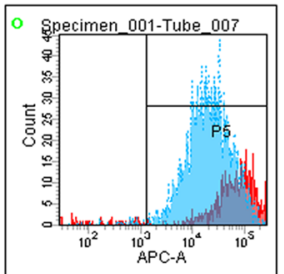

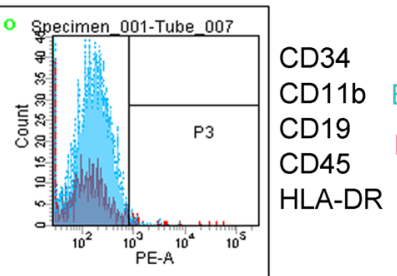

Blue $=$ Control siRNA BM-MSCs

Red=siGRP78 BM-MSCs

Fig. 2 Isolation and Characterization of siGRP78 modified MSCs derived from human bone marrow. a Flow cytometric analysis showed BM-MSCS were positive for mesenchymal lineage markers (CD73, CD90 and CD105), negative for hematopoietic and endothelial markers (CD34, CD11b, CD19, CD45), and negative for HLA-DR. b Representative morphology of BM-MSCS. c Down-regulating the expression of GRP78 in BM-MSCs do not influence the stemness trait of MSC. Blue was the control siRNA; red was the siGRP78. d qPCR showed GRP78 was down-regulated in the siGRP78 transfected BM-MSCs compared with control siRNA (scramble siRNA)

of Sorafenib. From the MTT assay, we found the IC50 of Sorafenib in HepG2 was about $10 \mu \mathrm{M}$, and PLC $12.5 \mu \mathrm{M}$, however the IC50 in HepG2-SR and PLC-SR cells was more than $20 \mu \mathrm{M}$ (Additional file 1: Figure S1A). Western blot showed GRP78 is overexpressed in SR cells. qPCR showed the same result with Western blot, GRP78 mRNA expressed higher in SR cells than in control cells. Therefore, we selected $10 \mu \mathrm{M}$ Sorafenib to treat HepG2 and $12.5 \mu \mathrm{M}$ Sorafenib to treat PLC cells in our further assays.

\section{Characterization of exosomes from siRNA against GRP78 modified BM-MSCs}

We transfected BM-MSCs with control siRNAs (scrambled siRNA) and siGRP78, exosomes were isolated from the conditioned medium $24 \mathrm{~h}$ after transfection and used for our further studies. To characterize the siGRP78 (siRNA against GRP78) modified exosomes, firstly, we examed the expression of exosomal markers [34]: Alix,
CD81 and CD63, which are all expressed in the modified exosomes (Fig. 2a). Then, we detected exosome size distribution (ranging between 4 and $120 \mathrm{~nm}$ ) by nanoparticle tracking analysis (NTA), and morphology by electron microscopy (EM) (Fig. 3b, c). By transmission electron microscopy, we determined BM-MSCs-derived exosomes were about 50 to $130 \mathrm{~nm}$ in width and physically homogeneous (Fig. 3c). Totally, the data suggest that exosome modification does not alter their size or surface markers. To quantify the loading efficiency of siGRP78 in exosomes from BM-MSCs, we used RT-PCR and found that $1 \%$ of the siRNA was retained in the exosomes after transfection.

To determine whether BM-MSCs-derived exosomes, either expressing siGRP78 or not, could be internalized by Sorafenib sensitive or resistant HCC cells, exosomes were labeled with the lipophilic dye PKH67. HepG2 and PLC cells, and their resistant cells (SR), treated at $37^{\circ} \mathrm{C}$ with $10 \mu \mathrm{g} / \mathrm{ml}$ of exosomes for $3 \mathrm{~h}$, internalized exosomes 


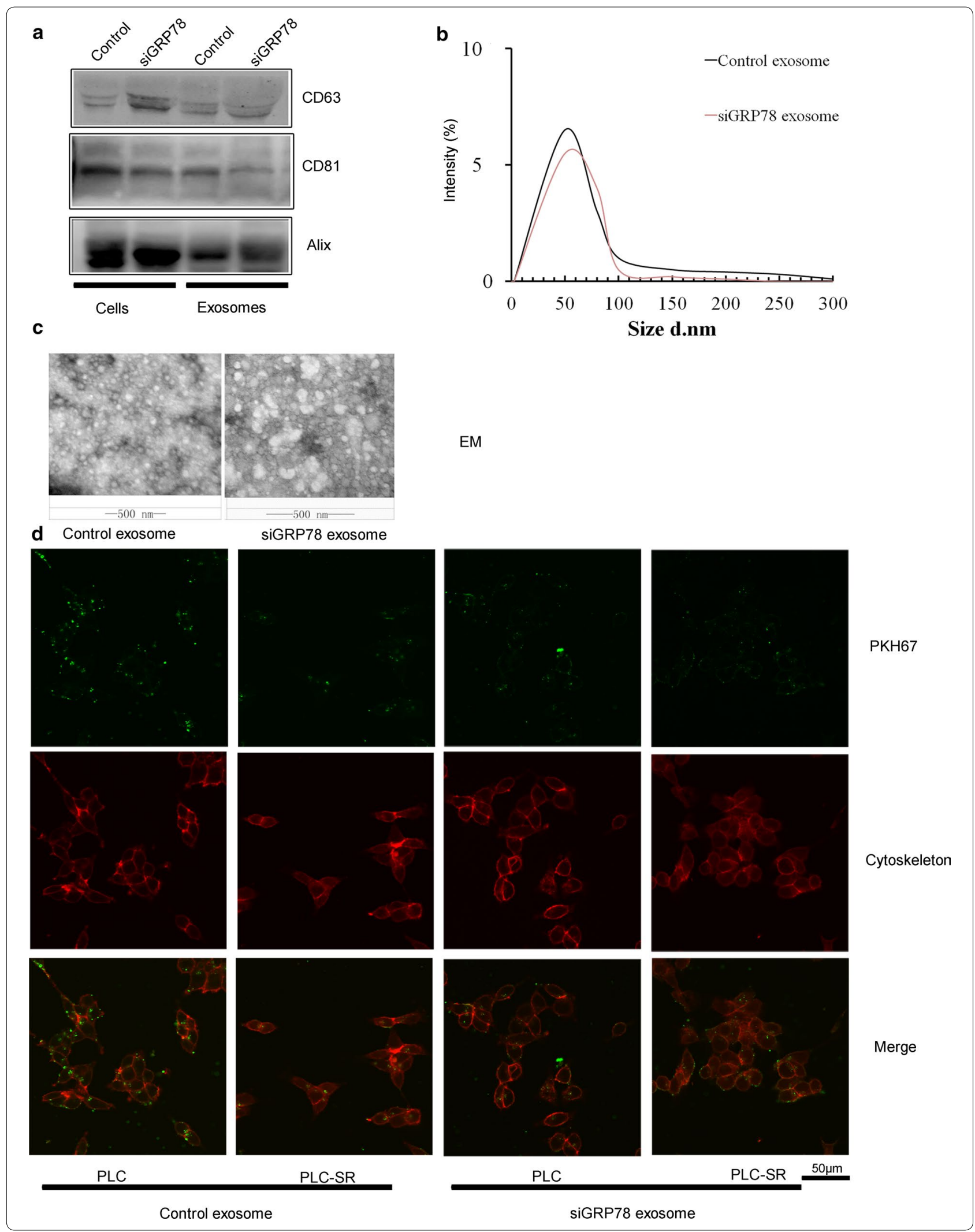


(See figure on previous page.)

Fig. 3 Generation and characterization of siGRP78 modified exosomes. a Western blot showed the exosomes from transfected or not with siGRP78. Protein levels of the two exosomes, CD63, CD81 and Alix were evaluated. b Exosome size distribution was determined by NTA. c Electron microscopy showed the morphology of BM-MSCs-derived exosomes. Scale bar $=500 \mathrm{~nm}$. d Confocal microscopy showed PLC cells treated with $10 \mathrm{\mu g} / \mathrm{ml}$ of control exosomes derived from BM-MSCs and siGRP78 modified exosomes from BM-MSCs. Cytoskeleton were stained with phalloidin-TRITC (red); exosomes were labeled with PKH67 (green). Scale bar $=50 \mu \mathrm{m}$

as shown in Fig. 3d and in Additional file 1: Figure S2. The results showed that siGRP78 modified exosomes could be internalized by all the cells. So, siGRP78 modified exosomes did not influence the internalization of exosomes.

\section{siGRP78 modified exosomes combined with Sorafenib inhibit the growth of HCC}

As lack of an appropriate delivery systems, the RNAbased therapy of HCC has been hampered in clinic. Here, we examined the possibility of loading exosomes with GRP78 specific siRNA to test their functional activity towards Sorafenib sensitive and resistant HCC cells. To test whether siGRP modified exosomes showed functional activity in inhibiting Sorafenib sensitive and resistant HCC cell growth, we treated Sorafenib-sensitive or resistant HepG2 or PLC cells for $48 \mathrm{~h}$ with $0.1,0.5$, 1 or $10 \mu \mathrm{g} / \mathrm{ml}$ of exosomes with scrambled siRNA or with siGRP78 and combined with or without Sorafenib (HepG2 was $10 \mu \mathrm{M}$, and PLC was $12.5 \mu \mathrm{M}$ ).

From Fig. 4a, we observed dose dependent reduced viability of the four cell lines treated with Sorafenib and siGRP78-modified exosomes $0.1,0.5,1$ or $10 \mu \mathrm{g} / \mathrm{ml}$ of exosomes $(P>0.05)$. As expected, Sorafenib treatment did not inhibit HepG2-SR (Sorafenib resistance) and PLC-SR cell growth. No differences were found in scrambled siRNA-modified exosomes compared to controls. siGRP78 modified exosomes inhibit the growth of sensitive and resistant HCC slightly. We also found that $10 \mu \mathrm{g} /$ $\mathrm{ml}$ of siGRP78-modified exosomes was the most effective concentration in HCC. So, we used $10 \mu \mathrm{g} / \mathrm{ml}$ of siGRP78modified exosomes for our further experiments.

To explore the relationship between anti-proliferative effect and GRP78 in Sorafenib resistance, Sorafenib sensitive or resistant cells treated with siGRP78-modified exosomes or scramble siRNA exosomes were added Sorafenib and subjected to immunoblotting and qPCR to detect the expression of GRP78. As shown in Fig. 4b, c, the treatment of HCC cells with siGRP78 modified exosomes was able to decrease the expression of GRP78 in all cells. And Sorafenib only inhibited GRP78 in sensitive HCC. In Sorafenib resistant HCC, Sorafenib could not inhibit GRP78 expression, inversely, promoted the mRNA expression of GRP78.

The ability of siGRP78 modified exosomes combined with Sorafenib to reduce HCC growth was also tested in an in vivo tumor xenograft model. PLC and PLC-SR cells $\left(1 \times 10^{7}\right)$ were inoculated subcutaneously in Balb/c nu/ nu mice; 1 week post cell injection, mice were injected around the tumor q.o.d with vehicle (PBS), Sorafenib $(25 \mathrm{mg} / \mathrm{kg})$ and $100 \mu \mathrm{g}$ of exosomes released by BMMSCs (Exo-scramble siRNA or Exo-siGRP78) with Sorafenib $(25 \mathrm{mg} / \mathrm{kg})$. After 1 month, mice were sacrificed and the tumors removed. Figure $4 \mathrm{~d}$ and Additional file 1: Figure S3 showed that tumor growth of PLC was reduced in mice treated with Sorafenib, and no tumor were found in mice treated with siGRP78 modified exosomes combined with Sorafenib. Correspondingly, in PLC-SR, obvious reduction in tumor size was observed in mice treated with siGRP78 modified exosomes combined with Sorafenib. There were no statistically significant differences between mice treated with control (scramble siRNA) exosomes combined with Sorafenib and Sorafenib treatment. Additional file 1: Figure S3 showed the final tumor weight of the tumors. Figure $4 \mathrm{e}$ showed that tumor growth of sensitive and resistant cells was reduced in mice treated with Sorafenib and siGRP78 modified exosomes. As expected, Sorafenib with or without scramble siRNA modified exosomes could not inhibit the tumor growth of resistant cancer cells. Compared with sensitive cells, Sorafenib resistant cells were resistant to Sorafenib.

Together, siGRP78 modified exosomes facilitated the sensitivity of HCC to Sorafenib and reversed the Sorafenib resistance in HCC.

\section{siGRP78 modified exosomes combined with Sorafenib inhibit the invasion of $\mathrm{HCC}$ in vitro}

To test whether siGRP modified exosomes could inhibit the invasion of Sorafenib sensitive and resistant HCC, we treated Sorafenib-sensitive or resistant HepG2 or PLC cells for $48 \mathrm{~h}$ with the four treatments (Control, Sorafenib, Sorafenib with scramble siRNA exosomes, Sorafenib with siGRP78 exosomes). The dose of Sorafenib uesd in HepG2 was $10 \mu \mathrm{M}$, and PLC was $12.5 \mu \mathrm{M}$. Then, we added the cells in the up layers. After $24 \mathrm{~h}$, we found Sorafenib could not inhibit the invasion of SR cells, and siGRP78 modified exosomes combined with Sorafenib inhibited the invasion of SR (Fig. 5a). No differences compared to Sorafenib were observed in scrambled siRNA modified exosomes. Figure $5 \mathrm{~b}$ showed the statistic analysis $\left({ }^{*} P<0.05\right)$. To show 

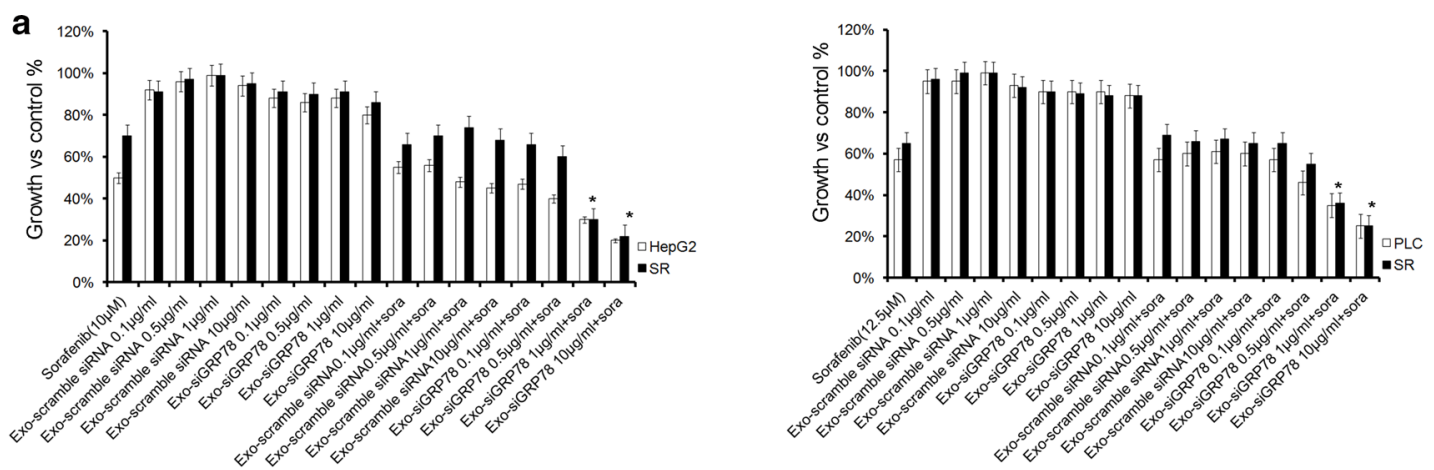

b
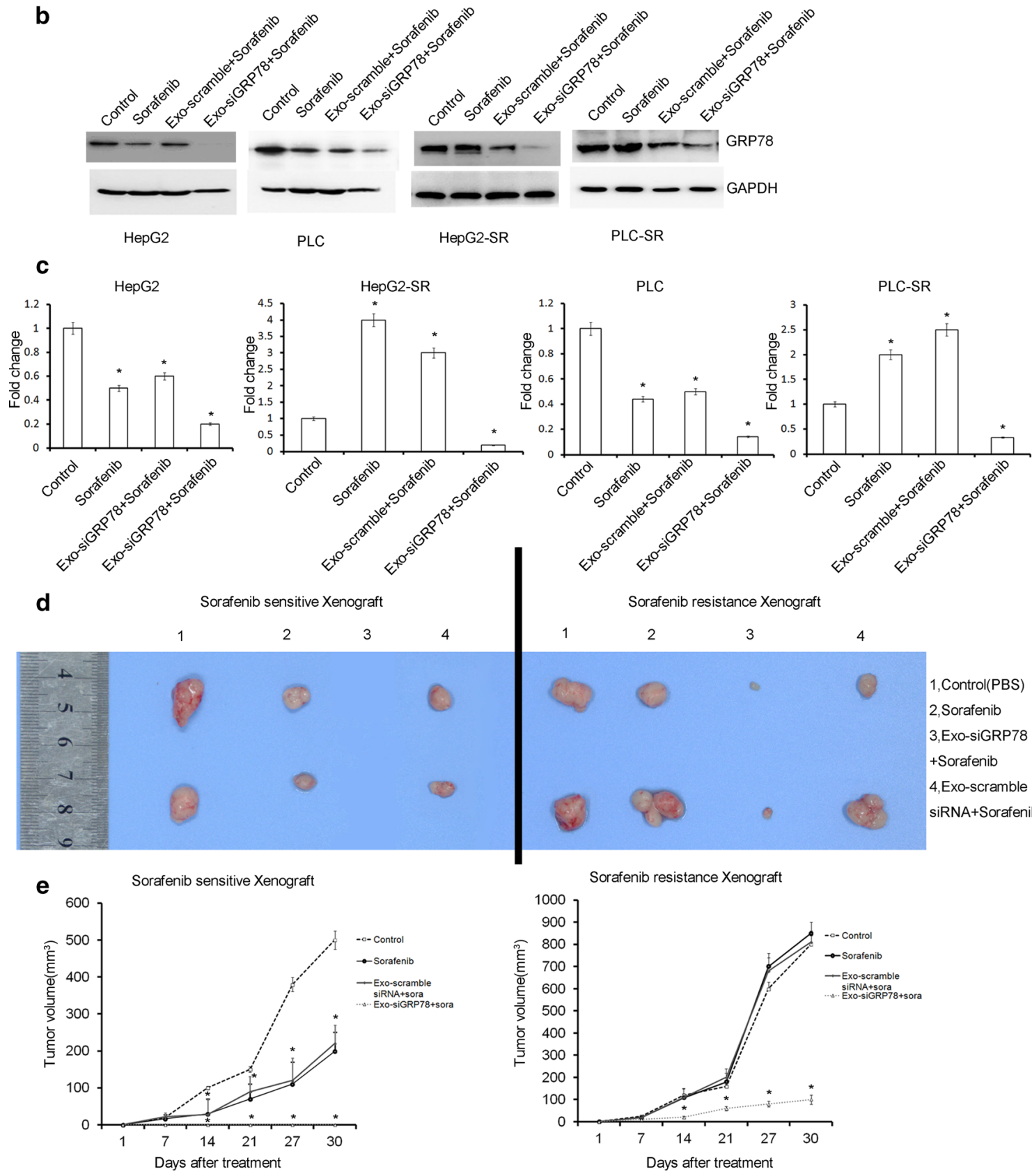

1,Control(PBS)

2,Sorafenib

3,Exo-siGRP78

+Sorafenib

4,Exo-scramble

siRNA+Sorafenib 
(See figure on previous page.)

Fig. 4 Effects of siGRP78 modified exosomes on the growth of Sorafenib sensitive and resistant cancer cells. a HepG2 (left panel) and PLC (right panel) growth was measured by MTT assay after $48 \mathrm{~h}$, (Sorafenib; 0.1 , 0.5, 1 or $10 \mu \mathrm{g} / \mathrm{ml}$ of Exo-scrambled siRNA or Exo-siGRP78 exosomes; and 0.1 , $0.5,1$ or $10 \mu \mathrm{g} / \mathrm{ml}$ of Exo-scrambled siRNA or Exo-siGRP78 modified exosomes combined with or without Sorafenib. The values were plotted as \% of growth vs Ctrl (untreated cells). Each point represents the mean \pm SEM of three independent experiments $\left({ }^{*} p<0.05\right.$, versus Sorafenib treatment). b Western blot analysis was performed on HepG2 and PLC cell lines, and their resistant cells treated for $48 \mathrm{~h}$ with Sorafenib, Sorafenib $+s c r a m b l e$ siRNA modified exosomes derived from BM-MSCs or siGRP78 modified exosomes + Sorafenib derived from BM-MSCs. Protein levels of GRP78 were evaluated. GAPDH as internal control. c qPCR showed the expression of GRP78 in HepG2 and PLC cell lines, and their resistant cells treated for $48 \mathrm{~h}$ with Sorafenib, Sorafenib + scramble siRNA modified exosomes or Sorafenib + siGRP78 modified exosomes derived from BM-MSCs. GAPDH as internal control. d The Subcutaneous orthotopic tumour growth in vivo assay showed the tumor size after different treatments (Control, Sorafenib, Exo-scramble siRNA + Sora and Exo-siGRP78 + Sora). Exo-siGRP78 + Sora significantly inhibited the the growth of sensitive or resistant cancer cells. (1) Control (PBS); (2) Sorafenib; (3) Exo-siGRP78 + Sora; (4) Exo-scramble siRNA + Sora, ( $n=5$ for each group, injections at 25 mg/ kg, q.o.d). e The median tumor volume showed the antitumor efficacy of the different treatments (Control, Sorafenib, Exo-scramble siRNA+ Sora and Exo-siGRP78 + Sora). Significant differences in terms of tumor volume were observed from day 14, Exo-siGRP78 + Sora versus Exo-scramble SiRNA + Sora $\left({ }^{*} P<0.05\right)$

the extracellular matrix degradation of HCC, we used zymography assay and found Sorafenib inhibited matrix degradation in sensitive cells. However, Sorafenib could not inhibit matrix degradation of resistant cells. siGRP78 modified exosomes combined with Sorafenib inhibited the ability of matrix degradation in sensitive and resistant cells (Fig. 5c). Figure $5 \mathrm{~d}$ also showed siGRP78 modified exosomes combined with Sorafenib inhibited the expression of MMP2 in sensitive and resistant cells. Correspondingly, Sorafenib could not inhibit the expression of MMP2 in resistant cells, and inhibited MMP2 in sensitive cells. The scramble siRNA exosomes could not sensitize SR cells to Sorafenib.

\section{siGRP78 modified exosomes combined with Sorafenib} inhibit the metastasis of Sorafenib resistant cells in vivo To investigate the role of siGRP78 modified exosomes on SR cells metastasis, HepG2-SR $\left(1 \times 10^{7} / 100 \mu \mathrm{l}\right)$ and PLC-SR $\left(1 \times 10^{7} / 100 \mu \mathrm{l}\right)$ cells were infected into the tail vein of Balb/c nu/nu mice. The 10 mice were randomly separated into 2 groups. One week after cell injection, mice were treated intraperitoneally q.o.d with vehicle (PBS), Sorafenib ( $25 \mathrm{mg} / \mathrm{kg}), 100 \mu \mathrm{g}$ of scramble siRNA modified exosomes + Sora and $100 \mu \mathrm{g}$ of siGRP78 modified exosomes + Sora. One month later, mice were sacrificed. We found that the treatment of mice bearing Sorafenib-resistant cells with siGRP78 modified exosomes determined less tumor metastasis in liver, compared to control mice (PBS) and to mice treated with scramble siRNA exosomes. No differences were observed in mice treated with exosomes containing scrambled siRNAs combined with Sorafenib compared to Sorafenib group (Fig. 6a). Figure 6b analyzed the number of tumor nodes in liver.

The data suggest that siGRP78 modified exosomes sensitize SR cells to Sorafenib in HCC metastasis.

\section{Discussion}

Although Sorafenib as tyrosine kinase inhibitor has revolutionized treatment and improved prognosis in HCC patients, the development of pharmacological resistance still remains a tricky problem [35]. One of the effective methods is to transfect a short interfering RNA (siRNA) to downregulate the expression of aberrant protein responsible for this drug resistance [19]. Although this approach is highly viable, it is difficult to find an effective target gene. Thereby, for clinical application of siRNAs, the stability and efficiency of delivery system is also a key challenge.

In this study, we propose a new approach to convey siRNA against GRP78, which plays vital roles in the process of Sorafenib resistance. BM-MSCs derived exosomes were used as a delivery system. BM-MSCs are a kind of adult stem cells, which have fine histocompatibility as tool cells. At present, the BM-MSCs are more and more popular in the therapy of diseases. Exosomes are natural carriers between cells in physiological state and also transfer chemotherapeutic drugs into tumors. Kim et al. used exosomes to deliver paclitaxel or doxorubicin in order to overcome multiple drug resistance in lung cancer.

Based on the previous researches, we tried to use siGRP78 modified exosomes from BM-MSCs to treat HepG2 and PLC cells or their resistant cells. First, we isolated BM-MSCs and transfected siGRP78 and scramble siRNA into the cells. And we found the exosomes could express siGRP78 and do not change the stemness of the BM-MSCs (Fig. 2). Then, we analyzed the exosomes from siGRP78 modified exosomes compared with scramble siRNA by western blot, NTA and EM, and demonstrated that the siGRP78 could not change the molecular markers, morphology and size of the exosomes of BM-MSCs (Fig. 3). In addition, confocal microscopy analysis showed that the sensitive 


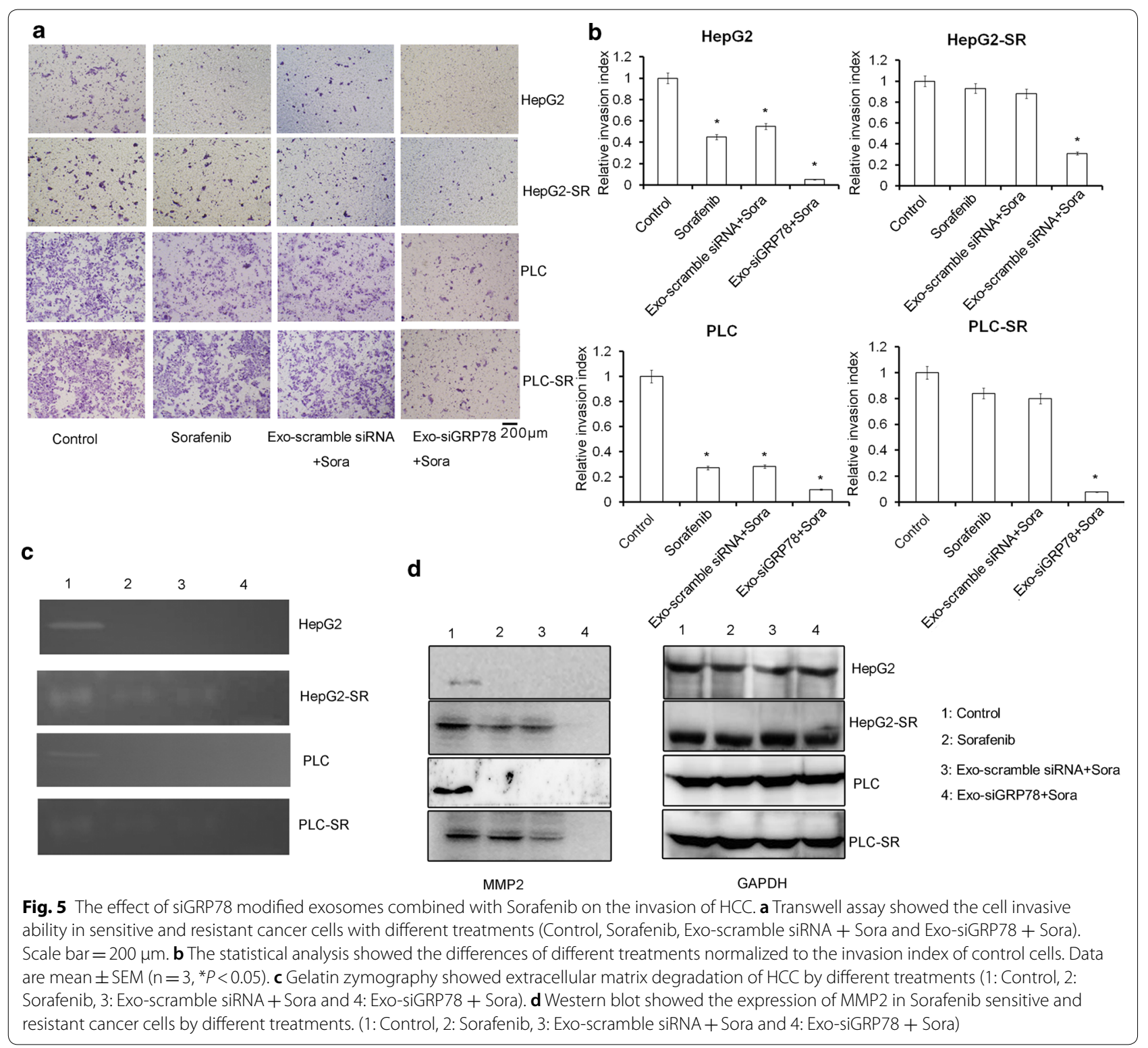

and resistant cancer cells internalize exosomes with or without siGRP78. This confirm the efficiency of siGRP78 or scramble siRNA delivery approach (Fig. 3d and Figure S2). It is possible to use exosomes in the delivery of siGRP78 to HCC cells. To demonstrate the effect of siGRP78 on the sensitive and resistant cancer cells, we employed MTT assay and found siGRP78 modified exosomes combined with Sorafenib inhibited the growth of both sensitive and resistant cancer cells. As expected, Sorafenib could inhibit the growth of sensitive cancer cells, but could not inhibit the growth of resistant cancer cells (Fig. 4a). To deeply explain GRP78 is involved in Sorafenib resistance, we detected the expression of GRP78 in all the cells of different treatments. And the results showed siGRP78 modified exosomes inhibit the expression of GRP78 in Sorafenib resistant cancer cells by Western blot. Also, in Sorafenib sensitive cancer cells, siGRP78 modified exosomes inhibited the expression of GRP78 (Fig. 4b). qPCR results showed the consistant results with Western blot (Fig. 4c). In vivo results showed the siGRP78 modified exosomes combined with Sorafenib obviously inhibit the growth of Sorafenib resistant cells. In Sorafenib sensitive cells group, there are no tumor growth (Fig. 4d, e). Finally, Transwell assay showed siGRP78 modified exosomes combined with Sorafenib inhibit the invasive ability. MMP2 and Zymography assay determined siGRP78 modified exosomes 


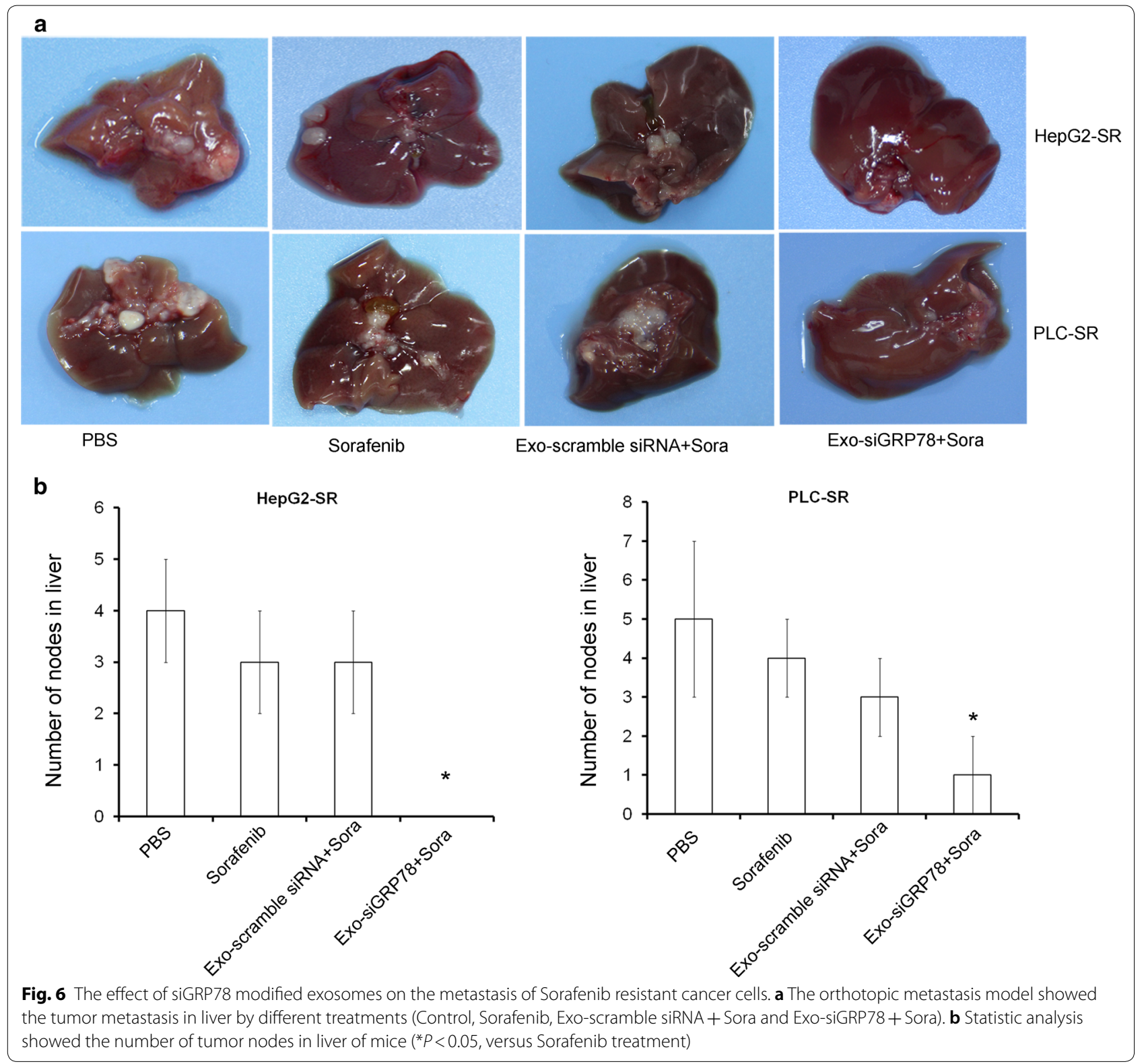

suppress the Matrix degradation (Fig. 5). In vivo metastasis model verified the conclusion of siGRP78 modified exosomes sensitize resistant cancer cells to SR (Fig. 6).

Taken together, our data are very promising and provide a rational base for the use of siGRP78 modified exosomes in a Sorafenib resistance therapy approach for use in HCC patients. Nevertheless, siGRP78 modified exosomes combined with Sorafenib could obviously inhibit sensitive HCC tumors. In clinics, we propose siGRP78 modified exosomes combined with Sorafenib together. While focusing on HCC, results from this study might have an impact on other types of tumors, such as colon cancer and gastric cancer, where GRP78 is abundantly expressed.

\section{Conclusion}

In conclusion, siGRP78 modified exosomes combined with Sorafenib are able to target GRP78 in hepatocellular carcinoma cells and inhibit the growth and invasion of the cancer cells in vitro. We demonstrated that exosomal transfer of siGRP78 enhanced chemosensitivity to Sorafenib in drug-resistant hepatocellular carcinoma. 


\section{Materials and methods Ethic approval}

This study was approval by the Ethic Committee at Jinzhou medical university. The use of the clinical specimens and animal for research purposes was in accordance with the Declaration of Helsinki.

\section{Isolation and characterization of human mesenchymal stem cells}

Bone marrow cells were isolated from femoral head after informed consent from patients undergoing hipreplacement surgery. The marrow were mixed with culture medium (MesenPRO $\mathrm{RS}^{\mathrm{TM}}$ Medium, Gibco, 12746-012) and isolated by h-BM-MSC isolation kit (TBD). The collected cells were plated in tissue culture flasks without further interference for 2-3 days. The culture medium was depleted by successive changes of culture medium (MesenPRO RS ${ }^{\mathrm{TM}}$ Medium, Gibco, 12746-012). A confluent monolayer culture with cells was observed 7 days following initial plating. Human BM-MSCs is characterized by the BD human MSC analysis kit (BD 562245).

\section{Cell culture}

The human Hepatocellular carcinoma cell line HepG2 and PLC were purchased from Cell bank of Chinese Academy of Sciences (Shanghai, PR China). All the cells were maintained in Dulbecco's modified Eagle's medium (DMEM, Gibco, Invitrogen, Carlsbad, CA) with $10 \%$ fetal bovine serum (FBS, Clark, Houston, TX, USA) and 1\% antibiotic-antimycotic solution (Life Technologies, NY, USA).

\section{Exosomes isolation}

Before exosomes collection, the BM-MSC were cultured in culture media containing centrifuged FBS, which was used to remove FBS-derived exosomes. During 24-48 h, the culture medium were collected and prepared for exosomes collection. Exosomes were collected from the medium of $50 \mathrm{ml}$ human BM-MSC cells. The culture media was placed on ice and centrifuged at $800 \mathrm{~g}$ for $10 \mathrm{~min}$ to sediment the cells and subsequently was centrifuged at $12,000 \mathrm{~g}$ for $20 \mathrm{~min}$ to remove the cellular debris. Exosomes were separated from the supernatant by centrifugation at $100,000 \mathrm{~g}$ for $2 \mathrm{~h}$. The exosome pellet was washed once in a large volume of PBS and re-suspended in $100 \mu \mathrm{l}$ of PBS (exosomes fraction).

\section{Exosome fluorescent labeling}

Exosomes were also isolated following the same procedure as described above, and for functional assays where exosomes were used, the concentration of total proteins contained in each exosomes pellet was quantified using the BCA assay. Exosomes were labeled with the green fluorescent linker PKH67 (Sigma-Aldrich), as the instruction showed. Briefly, bring the volume of the pellet sample up to $1 \mathrm{~mL}$ using Diluent $\mathrm{C}$ from the PKH67 kit. Add $6 \mu$ PKH67 dye into each of the $1 \mathrm{ml}$ Diluent $\mathrm{C}$ tubes, mix continuously for $30 \mathrm{~s}$ by gentle pipetting. Let stand at room temperature for 5 min. Quench by adding $2 \mathrm{ml} \mathrm{10 \%} \mathrm{BSA} \mathrm{in} \mathrm{PBS.} \mathrm{Bring}$ the volume up to $8.5 \mathrm{ml}$ in serum-free media. Make a $0.971 \mathrm{M}$ sucrose solution. Add $1.5 \mathrm{ml}$ of the sucrose solution by pipetting slowly and carefully into the bottom of your tube, making sure not to create turbulence. The exosomes-PKH67 solution will remain on top of a sucrose cushion. Centrifuge at $190,000 g$ for $2 \mathrm{~h}$ at $2-8{ }^{\circ} \mathrm{C}$. Resuspend the exosome pellet in $1 \times$ PBS by gentle pipetting.

\section{Electron microscopy}

Exosomes were adsorbed for $10 \mathrm{~min}$ to a carbon coated grid rendered hydrophilic and 20 min fixed with $4 \%$ paraformaldehyde, the excess liquid was removed with a filter paper, and samples were stained with $1 \%$ uranyl acetate for $30 \mathrm{~s}$. After excess uranyl formate was removed with a filter paper, grids were examined and images were recorded by transmission electron microscope (Japan, Hitachi 7650).

\section{Nanoparticle tracking analysis}

Particle size was measured by dynamic light scattering (Zetasizer Nano ZS; Malvern Instruments, Malvern, UK).

\section{siRNA transfection}

The siRNA sequences against Grp78 were designed by siRNA finder (Ambion, USA) and synthesized by Genechem Corporation (Shanghai, China). The sequences of sense strands of siRNA duplex were as follows: Grp78: 5'-AGACGCUGGAACUAUUGCUUU-3'. BM-MSCs were plated in six-well plate $\left(5 \times 10^{5}\right.$ cells/well), allowed to adhere for $24 \mathrm{~h}$ and transfected with siRNA. Transfection of siRNA was performed as lipofectamine 2000 Handbook (Invitrogen). Briefly, the cells were incubated for $4 \mathrm{~h}$ with the transfection complex containing $4 \mu \mathrm{g}$ siRNA. After $4 \mathrm{~h}$, the transfection complex was removed and the cells were incubated in complete growth medium for $48 \mathrm{~h}$. The transfection effect of siRNA was confirmed by qPCR and western blot.

\section{The preparation and quantification of the modified exosomes}

We prepared and quantified as Sander et al. described [36]. After transfection by siRNA or siRNA against 
GRP78, samples were diluted $10 \times$ with PBS and centrifuged at $100,000 \mathrm{~g}$ for $70 \mathrm{~min}$ to remove unbound siRNA. RNA was isolated from pellets with TRIzol Reagent according to manufacturer's recommendations. Reverse transcription of standards and samples was performed in a GeneAmp PCR System 9700 (Applied Biosystems, Foster City, CA) thermocycler using a TaqMan Reverse Transcription Kit, according to manufacturer's instructions. Each $7.5 \mu \mathrm{l}$ reverse transcription reaction contained $1 \mu \mathrm{l}$ of RNA template, $1 \mathrm{mM}$ dNTPs, $1.9 \mathrm{U}$ RNAse Inhibitor, $50 \mathrm{nM}$ reverse stem loop primer and $25 \mathrm{U}$ MultiScribe Reverse Transcriptase in $1 \times$ reverse transcription buffer.

Quantitative PCR was performed in $10 \mu \mathrm{l}$ reactions Amplification curves were analysed with Viia 7 software version 1.2.1. All samples for RT-PCR were prepared in triplicate and each RNA isolate was analysed in duplicate. Using this method, traces of siRNA could still be accurately quantified.

\section{Transwell assay}

Transwell assay was performed at Costar's 24 well Transwell (Costar \#3422). Cells were placed on 96-well-plate, at a concentration of $1 \times 10^{4} /$ well. After $24 \mathrm{~h}$, the inserts were inverted and stained with Crystal violet. The number of invade cells were observed and counted using fluorescent microscope. Five fields were randomly chosen and the numbers of penetrated cells were counted.

\section{MTT assay}

To explore the IC50 of HCC, The Hepatocellular carcinoma cells (HepG2 and PLC) were collected and replated into 96-well plate as 10,000 per well, then treated by Sorafenib $(0,2.5,5,10,15,20,25 \mu \mathrm{M})$.

To explore the effect of siGRP78 modified exosomes on HCC, we treated Sorafenib-sensitive or resistant HepG2 or PLC cells for $48 \mathrm{~h}$ with $0.1,0.5,1$ or $10 \mu \mathrm{g} / \mathrm{ml}$ of exosomes with scrambled siRNA or with siGRP78 and combined with or without Sorafenib, Sorafenib (HepG2 was about $10 \mu \mathrm{M}$, and PLC $12.5 \mu \mathrm{M}$ ).

Finally, we added each well with $20 \mu \mathrm{l}$ of MTT substrate for $4 \mathrm{~h}$; the medium was then removed and $100 \mu \mathrm{l}$ of DMSO was added. Plates were read at a wavelength of $490 \mathrm{~nm}$, with optical density (OD) reported normalized to blank wells containing only DMSO. We analyzed the relative growth rate as OD (treatment)/OD (control).

\section{Orthotopic tumour growth in Balb/c Nu Nu mice}

Female BALB/c nude mice were purchased from Beijing Vital River Laboratory Animal Technology Co., Ltd. All $\mathrm{BALB} / \mathrm{c}$ nude mice (4-5 weeks old, female) were maintained in SPF condition. Our animal experiments were approved by the Animal Care Committee.
Subcutaneous model: The 5-week-old BALB/c-nu mice were randomly divided into 4 groups ( $\mathrm{n}=5$ per group). HepG2, HepG2-SR (Sorafenib Resistance) and PLC and PLC-SR cancer cells $\left(1 \times 10^{7} / 0.1 \mathrm{ml}\right.$ PBS $)$ were inoculated subcutaneously into the left and right flank of the nude mice. After 1 week, we injected the drug around the tumors as day $7,9,11, \ldots$, when tumors were palpable, we treated the tumor with: (1) PBS (Ctrl), (2) Sorafenib (25 mg/kg), (3) Scramble siRNA modified exosomes from BM-MSCs combined with Sorafenib (Exo-scramble siRNA + Sora, $100 \mu \mathrm{g} /$ mouse + Sorafenib $25 \mathrm{mg} / \mathrm{kg}$ ), (4) siGRP78 modified exosomes derived exosomes (ExosiGRP78 + Sora, $100 \mu \mathrm{g} / \mathrm{mouse}+$ Sorafenib $25 \mathrm{mg} / \mathrm{kg}$ ).

Metastasis model: The 5-week-old BALB/c-nu mice were randomly divided into 4 groups $(n=5$ per group). To induce tumor metastasis, $1 \times 10^{7}$ tumor cells were injected into the tail vein of mice. After 1 week, mice were treated intraperitoneally q.o.d with: (1) PBS (Ctrl), (2) Sorafenib (25 mg/kg), (3) Scramble siRNA modified exosomes from BM-MSCs combined with Sorafenib (Exo- scramble siRNA + Sora, $100 \mu \mathrm{g} /$ mouse + Sorafenib $25 \mathrm{mg} / \mathrm{kg}$ ), (4) siGRP78 modified exosomes derived exosomes (Exo-siGRP78+Sora, $100 \mu \mathrm{g} /$ mouse + Sorafenib $25 \mathrm{mg} / \mathrm{kg}$ ).

The mice were sacrificed 30 days after inoculation and the tumors were analyzed by tumor weight.

\section{Western blot analysis}

For extraction of total cellular protein, cells were lysed in RIPA buffer with 1\% PMSF. Protein concentration was quantified using the BCA kit (Pierce Biotechnology, Inc., Rockford, IL). Proteins were analyzed by SDS-PAGE. The membranes were incubated overnight at $4{ }^{\circ} \mathrm{C}$ with the GRP78, MMP2 and GAPDH (1:1000) (Cell signaling technology, Danvas, MA). Thereafter, the membranes were incubated with HRP-labeled anti-rabbit secondary antibodies (1:1000) for $1 \mathrm{~h}$ at room temperature. At last, the membrane was visualized by enhanced chemiluminescence kit (Thermo Fisher Scientific Inc., Rockford, IL, USA).

\section{Gelatin zymography}

The Conditioned medium from the HCC cells was collected and concentrated at $2000 \mathrm{~g}, 10 \mathrm{~min}$. Equal amounts of protein were loaded and separated by $10 \%$ polyacrylamide gel containing $1 \mathrm{~g} / \mathrm{l}$ gelatin. The gels were re-natured in $2.5 \%$ Triton-X-100 with gentle agitation for $30 \mathrm{~min}$ at room temperature. The gel was pretreated by developing buffer $\left(5 \mathrm{mM} \mathrm{CaCl}_{2}, 50 \mathrm{mM}\right.$ Tris, and $0.2 \mathrm{mM} \mathrm{NaCl}$, $0.02 \%$ Brij35 ( $\mathrm{pH} 7.5)$ ) for $30 \mathrm{~min}$ at room temperature, then developed in developing buffer overnight at $37{ }^{\circ} \mathrm{C}$, stained with Coomassie Brilliant Blue R-250 for $30 \mathrm{~min}$ 
and destained with destaining solution. The protease activity was analyzed by gel imaging and analysis system.

\section{Quantitative real-time PCR assays}

mRNAs were isolated with TRIzol reagent (Invitrogen) and reverse transcribed. cDNAs were amplified by RTPCR. Expression assays were used to quantify the levels of different RNAs as follows:

GRP78(F:T TC AGC CAA T TA TCA GCA A AC TCT;R:TTT TCTGATGTATCC TCT TCACCAGT), GAPDH(F:TGTGGGCATCAATGGATTTGG;R:ACA CCATGTATTCCGGGTCAAT). Quantitative PCR was conducted in triplicate at $95^{\circ} \mathrm{C}$ for $10 \mathrm{~min}$, followed by 35 cycles of $95^{\circ} \mathrm{C}$ for $15 \mathrm{~s}$ and $60^{\circ} \mathrm{C}$ for $60 \mathrm{~s}$ (7300 Fast RealTime PCR System; Stratagen). Cycle thresholds were normalized to an internal control: U6 rRNA for precursor of miRNA and GAPDH for mRNA assays. The amount of RNA was calculated using the $2-{ }^{\Delta \Delta C T}$ method; the level of expression of RNA was normalized to the adapted internal control (denoted "relative expression") and, where appropriate, to the level of expression at Control (denoted "fold change").

\section{Statistical analysis}

All data were expressed as the mean \pm SEM. Comparisons between more than two groups were performed by one-way ANOVA. A P-value of $<0.05$ was considered statistically significant.

\section{Additional file}

Additional file 1: Figure S1. Characterization of Sorafenib resistant HCC cells. Figure S2. Characterization of exosomes from siRNA against GRP78 modified BM-MSCs. Figure S3. The final tumor weight of the tumors.

\section{Abbreviations}

BM-MSCs: bone marrow mesenchymal stem cells; HCC: hepatocellular carcinoma; Sora: Sorafenib; NTA: nanoparticle tracking analysis.

\section{Authors' contributions}

HL designed the study and performed the cell experiments; CY and YS performed the animal experiments and helped with the biological study; $\mathrm{HL}$ and LZ supervised the whole work and wrote the manuscript. All authors read and approved the final manuscript.

\section{Author details}

${ }^{1}$ Life Science Institute, Jinzhou Medical University, Jinzhou 121000, People's Republic of China. ${ }^{2}$ Department of General Surgery 2, Central Hospital of Jinzhou City, Jinzhou 121000, People's Republic of China. ${ }^{3}$ School of Pharmacy, Jinzhou Medical University, Jinzhou 121000, People's Republic of China.

\section{Acknowledgements}

This work was supported by the grants from National Natural Science Foundation of China (81502484).

\section{Competing interests}

The authors declare that they have no competing interests.

\section{Availability of data and materials}

Data sharing not applicable to this article as no datasets were generated or analyzed during the current study.

\section{Ethics approval and consent to participate}

This study was approval by the Ethic Committee at Jinzhou medical university. The use of the clinical specimens and animal for research purposes was in accordance with the Declaration of Helsinki, and the research didn't contain any individual person's data in any form.

\section{Funding}

This work was supported by the National Natural Science Foundation of China (81502484) in the design of the study and analysis of data.

\section{Publisher's Note}

Springer Nature remains neutral with regard to jurisdictional claims in published maps and institutional affiliations.

Received: 7 September 2018 Accepted: 7 December 2018

Published online: 20 December 2018

\section{References}

1. Forner A, Llovet JM, Bruix J. Hepatocellular carcinoma. Lancet. 2012;379:1245-55.

2. Maluccio M, Covey A. Recent progress in understanding, diagnosing, and treating hepatocellular carcinoma. CA. 2012;62:394-9.

3. Forner A. Hepatocellular carcinoma surveillance with miRNAs. Lancet Oncol. 2015;16:743-5.

4. Rahmani M, Davis EM, Crabtree TR, Habibi JR, Nguyen TK, Dent P, et al. The kinase inhibitor sorafenib induces cell death through a process involving induction of endoplasmic reticulum stress. Mol Cell Biol. 2007;27:5499-513.

5. Li R, Yanjiao G, Wubin H, Yue W, Jianhua H, Huachuan Z, et al. Secreted GRP78 activates EGFR-SRC-STAT3 signaling and confers the resistance to sorafeinib in HCC cells. Oncotarget. 2017;8:19354-64.

6. Bruix J, Takayama T, Mazzaferro V, Chau GY, Yang J, Kudo M, et al. Adjuvant sorafenib for hepatocellular carcinoma after resection or ablation (STORM): a phase 3, randomised, double-blind, placebo-controlled trial. Lancet Oncol. 2015;16:1344-54.

7. $\mathrm{Wu} \mathrm{CH}$, Wu X, Zhang HW. Inhibition of acquired-resistance hepatocellular carcinoma cell growth by combining sorafenib with phosphoinositide 3-kinase and rat sarcoma inhibitor. J Surg Res. 2016;206:371-9.

8. Kuczynski EA, Lee CR, Man S, Chen E, Kerbel RS. Effects of sorafenib dose on acquired reversible resistance and toxicity in hepatocellular carcinoma. Can Res. 2015;75:2510-9.

9. Xing $X$, Lai $M$, Wang $Y, X u E$, Huang Q. Overexpression of glucose-regulated protein 78 in colon cancer. Clin Chim Acta. 2006;364:308-15.

10. Kwon D, Koh J, Kim S, Go H, Min HS, Kim YA, et al. Overexpression of endoplasmic reticulum stress-related proteins, XBP1s and GRP78, predicts poor prognosis in pulmonary adenocarcinoma. Lung cancer. 2018:122:131-7.

11. Zheng HC, Takahashi H, Li XH, Hara T, Masuda S, Guan YF, et al. Overexpression of GRP78 and GRP94 are markers for aggressive behavior and poor prognosis in gastric carcinomas. Hum Pathol. 2008;39:1042-9.

12. Yeung BH, Kwan BW, He QY, Lee AS, Liu J, Wong AS. Glucose-regulated protein 78 as a novel effector of BRCA1 for inhibiting stress-induced apoptosis. Oncogene. 2008;27:6782-9.

13. Luo C, Xiong H, Chen L, Liu X, Zou S, Guan J, et al. GRP78 promotes hepatocellular carcinoma proliferation by increasing FAT10 expression through the NF-kappaB pathway. Exp Cell Res. 2018;365:1-11.

14. Chiou JF, Tai CJ, Huang MT, Wei PL, Wang YH, An J, et al. Glucose-regulated protein 78 is a novel contributor to acquisition of resistance to sorafenib in hepatocellular carcinoma. Ann Surg Oncol. 2010;17:603-12.

15. Yu M, Han S, Kou Z, Dai J, Liu J, Wei C, et al. Lipid nanoparticle-based co-delivery of epirubicin and BCL-2 siRNA for enhanced intracellular drug release and reversing multidrug resistance. Artif Cells Nanomed Biotechnol. 2018;46:323-32. 
16. Yu C, Ding B, Zhang X, Deng X, Deng K, Cheng Z, et al. Targeted iron nanoparticles with platinum-(IV) prodrugs and anti-EZH2 siRNA show great synergy in combating drug resistance in vitro and in vivo. Biomaterials. 2018;155:112-23.

17. Lin $G$, Zhu W, Yang L, Wu J, Lin B, Xu Y, et al. Delivery of siRNA by MRIvisible nanovehicles to overcome drug resistance in MCF-7/ADR human breast cancer cells. Biomaterials. 2014;35:9495-507.

18. Meng H, Liong M, Xia T, Li Z, Ji Z, Zink Jl, et al. Engineered design of mesoporous silica nanoparticles to deliver doxorubicin and P-glycoprotein siRNA to overcome drug resistance in a cancer cell line. ACS Nano. 2010;4:4539-50

19. Meng H, Mai WX, Zhang H, Xue M, Xia T, Lin S, et al. Codelivery of an optimal drug/siRNA combination using mesoporous silica nanoparticles to overcome drug resistance in breast cancer in vitro and in vivo. ACS Nano. 2013;7:994-1005.

20. Bellavia D, Raimondo S, Calabrese G, Forte S, Cristaldi M, Patinella A, et al. Interleukin 3-receptor targeted exosomes inhibit in vitro and in vivo chronic myelogenous leukemia cell growth. Theranostics. 2017;7:1333-45

21. Cheng X, Zhang G, Zhang L, Hu Y, Zhang K, Sun X, et al. Mesenchymal stem cells deliver exogenous miR-21 via exosomes to inhibit nucleus pulposus cell apoptosis and reduce intervertebral disc degeneration. J Cell Mol Med. 2018;22:261-76.

22. Corrado C, Raimondo S, Chiesi A, Ciccia F, De Leo G, Alessandro R. Exosomes as intercellular signaling organelles involved in health and disease: basic science and clinical applications. Int J Mol Sci. 2013;14:5338-66.

23. Simons M, Raposo G. Exosomes-vesicular carriers for intercellular communication. Curr Opin Cell Biol. 2009;21:575-81.

24. Conigliaro A, Costa V, Lo Dico A, Saieva L, Buccheri S, Dieli F, et al. CD90+ liver cancer cells modulate endothelial cell phenotype through the release of exosomes containing H19 IncRNA. Mol Cancer. 2015;14:155.

25. Koldehoff M, Zakrzewski JL, Beelen DW, Elmaagacli AH. Additive antileukemia effects by GFI1B- and BCR-ABL-specific siRNA in advanced phase chronic myeloid leukemic cells. Cancer Gene Ther. 2013;20:421-7.

26. Haney MJ, Klyachko NL, Zhao Y, Gupta R, Plotnikova EG, He Z, et al. Exosomes as drug delivery vehicles for Parkinson's disease therapy. J Controll Release. 2015;207:18-30.
27. El Andaloussi S, Lakhal S, Mager I, Wood MJ. Exosomes for targeted siRNA delivery across biological barriers. Adv Drug Deliv Rev. 2013;65:391-7.

28. Johnsen KB, Gudbergsson JM, Skov MN, Pilgaard L, Moos T, Duroux M A comprehensive overview of exosomes as drug delivery vehiclesendogenous nanocarriers for targeted cancer therapy. Biochem Biophys Acta. 2014;1846:75-87.

29. Nojehdehi S, Soudi S, Hesampour A, Rasouli S, Soleimani M, Hashemi SM. Immunomodulatory effects of mesenchymal stem cell-derived exosomes on experimental type-1 autoimmune diabetes. J Cell Biochem. 2018;119(11):9433-43.

30. Zhang S, Chuah SJ, Lai RC, Hui JHP, Lim SK, Toh WS. MSC exosomes mediate cartilage repair by enhancing proliferation, attenuating apoptosis and modulating immune reactivity. Biomaterials. 2018;156:16-27.

31. Chen J, Chopp M. Exosome therapy for stroke. Stroke. 2018;49:1083-90.

32. Ren J, Ding L, Zhang D, Shi G, Xu Q, Shen S, et al. Carcinoma-associated fibroblasts promote the stemness and chemoresistance of colorectal cancer by transferring exosomal IncRNA H19. Theranostics. 2018;8:3932-48.

33. Ono M, Kosaka N, Tominaga N, Yoshioka Y, Takeshita F, Takahashi RU, et al. Exosomes from bone marrow mesenchymal stem cells contain a microRNA that promotes dormancy in metastatic breast cancer cells. Sci signal. 2014;7:ra63.

34. Du Y, Li D, Han C, Wu H, Xu L, Zhang M, et al. Exosomes from humaninduced pluripotent stem cell-derived mesenchymal stromal cells (hiPSC-MSCs) protect liver against hepatic ischemia/reperfusion injury via activating sphingosine kinase and sphingosine-1-phosphate signaling pathway. Cell Physiol Biochem. 2017;43:611-25.

35. Zhang Y, Li D, Jiang Q, Cao S, Sun H, Chai Y, et al. Novel ADAM-17 inhibitor ZLDI-8 enhances the in vitro and in vivo chemotherapeutic effects of Sorafenib on hepatocellular carcinoma cells. Cell Death Dis. 2018;9:743.

36. Kooijmans SAA, Stremersch S, Braeckmans K, de Smedt SC, Hendrix A, Wood MJA, et al. Electroporation-induced siRNA precipitation obscures the efficiency of siRNA loading into extracellular vesicles. J Controll Release. 2013;172:229-38.
Ready to submit your research? Choose BMC and benefit from:

- fast, convenient online submission

- thorough peer review by experienced researchers in your field

- rapid publication on acceptance

- support for research data, including large and complex data types

- gold Open Access which fosters wider collaboration and increased citations

- maximum visibility for your research: over $100 \mathrm{M}$ website views per year

At $\mathrm{BMC}$, research is always in progress.

Learn more biomedcentral.com/submissions 